\title{
Moment-Guided Discriminative Manifold Correlation Learning on Ordinal Data
}

DOI:

$10.1145 / 3402445$

\section{Document Version}

Accepted author manuscript

Link to publication record in Manchester Research Explorer

\section{Citation for published version (APA):}

Tian, Q., Zhang, W., Cao, M., Wang, L., Chen, S., \& Yin, H. (2020). Moment-Guided Discriminative Manifold Correlation Learning on Ordinal Data. ACM Transactions on Intelligent Systems and Technology, 11(5), 1-18. https://doi.org/10.1145/3402445

\section{Published in:}

ACM Transactions on Intelligent Systems and Technology

\section{Citing this paper}

Please note that where the full-text provided on Manchester Research Explorer is the Author Accepted Manuscript or Proof version this may differ from the final Published version. If citing, it is advised that you check and use the publisher's definitive version.

\section{General rights}

Copyright and moral rights for the publications made accessible in the Research Explorer are retained by the authors and/or other copyright owners and it is a condition of accessing publications that users recognise and abide by the legal requirements associated with these rights.

\section{Takedown policy}

If you believe that this document breaches copyright please refer to the University of Manchester's Takedown Procedures [http://man.ac.uk/04Y6Bo] or contact uml.scholarlycommunications@manchester.ac.uk providing relevant details, so we can investigate your claim.

\section{OPEN ACCESS}




\title{
Moment-Guided Discriminative Manifold Correlation Learning On Ordinal Data
}

\author{
QING TIAN, WENQIANG ZHANG and MENG CAO, Nanjing University of Information Science \\ and Technology \\ LIPING WANG and SONGCAN CHEN, Nanjing University of Aeronautics and Astronautics \\ HUJUN YIN, The University of Manchester
}

\begin{abstract}
Canonical correlation analysis (CCA) is a typical and useful learning paradigm in big data analysis for capturing correlation across multiple views of the same objects. When dealing with such data with additional ordinal information, traditional CCA suffers from poor performance due to ignoring the ordinal relationships within the data. Such data is becoming increasingly common as either temporal or sequential information is often associated with the data collection process. In order to incorporate the ordinal information into the objective function of CCA, the so-called ordinal discriminative CCA (OR-DisCCA) has been presented in the literature. Although OR-DisCCA can yield better ordinal regression results, its performance deteriorates when the data is corrupted with noise and outliers, as they tend to smear the order information contained in class centers. To address this issue, in this paper we construct a robust, manifold-preserved ordinal discriminative correlation regression (rmODCR). The robustness is achieved by replacing the traditional ( $l_{2}$-norm) class centers with $l_{p}$-norm centers where $p$ is efficiently estimated according to the moments of the data distributions, as well as by incorporating the manifold distribution information of the data in the objective optimization. In addition, we further extend rmODCR with deep convolutional architectures. Extensive experimental evaluations have demonstrated the superiority of the proposed method.
\end{abstract}

CCS Concepts: $\bullet$ Computing methodologies $\rightarrow$ Artificial intelligence; Machine learning; Machine learning approaches;

Additional Key Words and Phrases: Canonical correlation analysis; Ordinal regression; $l_{p}$-norm centers; Manifold learning; Moment

ACM Reference Format:

Qing Tian, Wenqiang Zhang, Meng Cao, Liping Wang, Songcan Chen, and Hujun Yin, 2019. Moment-Guided Discriminative Manifold Correlation Learning On Ordinal Data. ACM Trans. Intell. Syst. Technol. 9, 4, Article 39 (March 2010), 19 pages.

DOI : 0000001.0000001

This work was partially supported by the National Natural Science Foundation of China under grant 61702273, the Natural Science Foundation of Jiangsu Province under grant BK20170956, the Natural Science Foundation of the Jiangsu Higher Education Institutions of China under grant 17KJB520022, the Fundamental Research Funds for the Central Universities No. NJ2019010, the Project Funded by the Priority Academic Program Development of Jiangsu Higher Education Institutions, the Priority Academic Program Development of Jiangsu Higher Education Institutions (PAPD) fund, and the Startup Foundation for Talents of Nanjing University of Information Science and Technology.

Author's addresses: Qing Tian, Wenqiang Zhang and Meng Cao are with the School of Computer and Software, Nanjing University of Information Science and Technology (Wenqiang Zhang and Meng Cao are co-second authors, E-mail: tianqing@nuist.edu.cn; cnzhangwenqiang@gmail.com; alrash@nuist.edu.cn). Qing Tian is also with the Collaborative Innovation Center of Atmospheric Environment and Equipment Technology, Nanjing University of Information Science and Technology, Nanjing, China, and the MIIT Key Laboratory of Pattern Analysis and Machine Intelligence, Nanjing University of Aeronautics and Astronautics, Nanjing, China. Liping Wang is with the Department of Mathematics, Nanjing University of Aeronautics and Astronautics, China (E-mail: wlpmath@nuaa.edu.cn). Songcan Chen (corresponding author) is with the College of Computer Science and Technology, Nanjing University of Aeronautics and Astronautics, China (E-mail: s.chen@nuaa.edu.cn). Hujun Yin is with the School of Electrical and Electronic Engineering, The University of Manchester, UK (E-mail: hujun.yin@manchester.ac.uk).

Permission to make digital or hard copies of part or all of this work for personal or classroom use is granted without fee provided that copies are not made or distributed for profit or commercial advantage and that copies bear this notice and the full citation on the first page. Copyrights for third-party components of this work must be honored. For all other uses, contact the owner/author(s).

(c) 2010 Copyright held by the owner/author(s). 2157-6904/2010/03-ART39 $\$ 15.00$

DOI : 0000001.0000001 


\section{INTRODUCTION}

In the emerging data science, with multiple sensory measurements being taken in increasingly number of applications in such as security surveillance, medical or health records, product surveys and corpus of online documents, it is important to discover association or extract correlation among these different representatives or views. Canonical correlation analysis (CCA) was first proposed in 1936 [Pearson 1936] to find projection vectors along which the correlation of two sets of variables is mutually maximized. However, the original CCA can only handle data with two views of variables. In order to analyze data with multiple views, Ketterling generated a multi-view counterpart [Kettenring 1971]. Motivated by this work, a variety of variants of CCA have been successively proposed, such as, kernel CCA [Melzer et al. 2003] [Lai and Fyfe 2000], sparse CCA [Parkhomenko 2008] [David and John Shawe 2011], robust and sparse CCA [Shi et al. 2017], probabilistic CCA [Kamada et al. 2015], and architecture-deepened CCA [Andrew et al. 2013]. The aforementioned methods are unsupervised as the label information is not incorporated in their objectives. To overcome this drawback and to extend the projection for classification, supervised discriminative counterpart of CCA has been proposed by minimizing the inter-class cross-view correlation while maximizing the intra-class cross-view correlation in the objective function [Sun et al. 2008b], [Sun et al. 2008a]. Furthermore, semi-supervised [Hou et al. 2010], sparse unsupervised [Han et al. 2012] and spectral embedding [Feng et al. 2017] based multi-view learning have also been introduced in CCA learning. The above reviewed CCA and variants have found wide applications in image processing [Ji et al. 2015] [Huang et al. 2010], multimedia analysis [Sargin et al. 2006] [Sargin et al. 2007] [Tae-Kyun and Roberto 2009], information retrieval [Vinokourov et al. 2002], text classification [Rupnik and Grobelnik 2008], biometric recognition [Zhai et al. 2012] [Haghighat et al. 2016] [Xie et al. 2016], human mobility modeling [Zhang et al. 2017], etc.

Although the above methods can yield promising results on their concerned problem$\mathrm{s}$, their performances dramatically deteriorate when dealing with ordinal data whose ground-truth labels are discrete and orderly monotonous. This is because that the ordinality characteristic of the data labels or sequences is not taken into account in the learning process. For the sake of clarification, we take age estimation as an example, which is a typical ordinal estimation problem. Age 16 is much closer to age 15 than to age 20 , and the severity of misclassifying the face aged 16 to age 15 is less than to age 20. But, if we disregard such an ordinal relationship and assign them with same severity weight, the estimation can be seriously unreliable. To address such ordinal data tasks with multi-view feature representations, Kawashima et al. [Kawashima et al. 2013] proposed to first perform multi-view fusion by CCA and then to conduct ordinal regression in the fused space. Although the ordinality relationship of data labels was specially preserved, the multi-view fusion and subsequent decision making were performed in a separate manner, leading to non-optimal performance. To incorporate both discriminative and ordinality information in the CCA objective function, Zhou et al. [Zhou and Chen 2014] proposed the so-called ordinal discriminative CCA (i.e. OR-DisCCA) by explicitly modeling both supervised label information and ordinality relationships between class labels. Although the OR-DisCCA method generated comparatively better regression results, its performance will be degenerated if the data is corrupted with outliers. Through analyzing the objective function of the OR-DisCCA, we find that the ordinal constraints are imposed on the class centers derived from $l_{2}$ norm, which is sensitive to outliers [Kloft et al. 2009] [Nie et al. 2010] [Kloft et al. 2011]. As a consequence, when data is corrupted with outliers or contains noise samples (in real applications, the data is frequently corrupted with outlier samples), these $l_{2}$-norm centers will be biased by them and thus the subsequent ordinal regression 
accuracy will be reduced. In addition, the discriminative within-class scatters incorporated in the objective function can also be easily biased by the outliers far from the true distributions. To overcome these drawbacks, with similar motivations to that of [Wang and Chen 2017], [Tian et al. 2018], we in this paper propose to replace the traditional $l_{2}$-norm centers with robust $l_{p}$-norm centers where the parameter $p$ is efficiently estimated according to the moments of the data distributions. Besides, to preserve the ordinal data spatial relationships, we construct a supervised ordinal manifold structure regularizer for better generalization performance. By taking into account both the above two considerations, we consequently generate a robust manifold ordinal discriminative correlation regression (rmODCR), whose efficacy and superiority is evaluated by extensive experiments. In addition, to improve the nonlinearly-discriminating performance of rmODCR, we further extend it with deep convolutional architectures. It is worth noting that although the concept of $l_{p}$-norm center has been presented in our previous work [Tian et al. 2018], there are substantial distinctions between them. First, the work [Tian et al. 2018] just concentrated on single view tasks while in this work we generate models in multi-view scenarios, which is not a ready extension of [Tian et al. 2018]. Second, the parameter $p$ in [Tian et al. 2018] was tuned through labor-intensive cross-validation. By contrast, in this work we analytically evaluate $p$ directly according to the moments of the data distributions, which is more computationally efficient. The main contributions of this paper are four-fold as follows:

(1) Proposing a type of outliers-insensitive multi-view $l_{p}$-norm class center algorithm with the parameter $p$ adaptively estimated according to the moments of the data distributions, in either joint or separate manner.

(2) Constructing a supervised ordinal manifold regularizer to alleviate the undesirable influence to the data distributions caused by the outliers.

(3) Building a robust manifold ordinal discriminative correlation regression ( $\mathrm{rmOD}$ CR) by incorporating the above two factors and extending it with deep convolutional architectures.

(4) Evaluating the efficacy and superiority of the proposed methodologies through extensive experiments.

The rest of this paper is organized as follows. Section 2 introduces related works. In Section 3, we elaborate the proposed models and corresponding algorithms. In Section 4 , experiments results and analysis are reported. Finally, Section 5 concludes this work and provides future directions.

\section{RELATED WORKS}

\subsection{CCA}

CCA [Pearson 1936] is one of the most typical cross-view correlation analysis tools. Specifically, for $N$ two-view training samples, represented by $\mathbf{X}=\left[\mathbf{x}_{1}, \cdots, \mathbf{x}_{N}\right] \in \mathbb{R}^{p \times N}$ and $\mathbf{Y}=\left[\mathbf{y}_{1}, \cdots, \mathbf{y}_{N}\right] \in \mathbb{R}^{q \times N}$, the conventional CCA algorithm seeks two projections $\mathbf{w}_{x} \in \mathbb{R}^{p}$ and $\mathbf{w}_{y} \in \mathbb{R}^{q}$, such that the correlation between $\mathbf{w}_{x}^{T} \mathbf{X}$ and $\mathbf{w}_{y}^{T} \mathbf{Y}$ is maximum

$$
\max _{\left\{\mathbf{w}_{x}^{T} \mathbf{w}_{x}=1, \mathbf{w}_{y}^{T} \mathbf{w}_{y}=1\right\}} \frac{\mathbf{w}_{x}^{T} \mathbf{X} \mathbf{Y}^{T} \mathbf{w}_{y}}{\sqrt{\mathbf{w}_{x}^{T} \mathbf{X} \mathbf{X}^{T} \mathbf{w}_{x} \mathbf{w}_{y}^{T} \mathbf{Y} \mathbf{Y}^{T} \mathbf{w}_{y}}} .
$$

where $\mathbf{w}_{x}$ and $\mathbf{w}_{y}$ respectively denote the projections for two views of training data. The numerator term $\mathbf{w}_{x}^{T} \mathbf{X Y}^{T} \mathbf{w}_{y}$ models the correlations between projected two views, while the denominator restricts the scatters for each view. Along the line of generalized multivariate linear regression theory [Hoegaerts et al. 2005], (1) can be alternatively 
formulated as

$$
\begin{aligned}
& \min _{\left\{\mathbf{w}_{x}, \mathbf{w}_{y}\right\}}\left\|\mathbf{w}_{x}^{T} \mathbf{X}-\mathbf{w}_{y}^{T} \mathbf{Y}\right\|_{2}^{2} \\
& \text { s.t. } \quad \mathbf{w}_{x}^{T} \mathbf{X} \mathbf{X}^{T} \mathbf{w}_{x}=1, \mathbf{w}_{y}^{T} \mathbf{Y} \mathbf{Y}^{T} \mathbf{w}_{y}=1 .
\end{aligned}
$$

After obtaining $\mathbf{w}_{x}$ and $\mathbf{w}_{y}$, we can make decisions for test instances by using their projected view representations.

\subsection{OR-DisCCA}

CCA is originally designed for analyzing ordinary tasks, its performance usually gets worse when faced with ordinal data in which there exist discrete order relationships among the data labels. Let us take human age labels as an example, 10-year is elder than 8-year, but younger than 12-year. To preserve such ordinality, an ordinal extension of CCA, called OR-DisCCA [Zhou and Chen 2014], was proposed to address such ordinal correlation regression tasks, whose objective function is formulated as

$$
\begin{array}{ll}
\min _{\{\mathbf{w}, \rho\}} & \left\|\mathbf{w}_{x D}^{T} \mathbf{X}-\mathbf{w}_{y D}^{T} \mathbf{Y}\right\|_{2}^{2}+\lambda_{1} \mathbf{w}_{D}^{T} \mathbf{S}_{w} \mathbf{w}_{D}-\lambda_{2} \rho+\lambda_{3} \sum_{d=1}^{D-1}\left(\mathbf{w}_{d}^{T} \mathbf{w}_{D}\right)^{2} \\
\text { s.t. } \quad \mathbf{w}_{D}^{T}\left(\mathbf{m}_{k+1}-\mathbf{m}_{k}\right) \geq \rho, k=1, \ldots, K-1,
\end{array}
$$

where $\mathbf{w}_{D}=\left[\mathbf{w}_{x D}^{T}, \mathbf{w}_{y D}^{T}\right]^{T}$ and $\mathbf{w}_{d}=\left[\mathbf{w}_{x d}^{T}, \mathbf{w}_{y d}^{T}\right]^{T}$ respectively indicating the $D$ thdirectional and $d$ th-directional cross-view projections, $\mathbf{m}_{k}=\left[\mathbf{m}_{x, k}^{T}, \mathbf{m}_{y, k}^{T}\right]^{T}$ with $\mathbf{m}_{x, k}=$ $\frac{1}{N_{k}} \sum_{\mathbf{x} \in \mathbf{X}_{k}} \mathbf{x}$ and $\mathbf{m}_{y, k}=\frac{1}{N_{k}} \sum_{\mathbf{y} \in \mathbf{Y}_{k}} \mathbf{y}$ respectively denoting the $k$ th class centers of $\mathbf{X}$ and Y. $\mathbf{S}_{w}=\frac{1}{N} \sum_{k=1}^{K} \sum_{\mathbf{x} \in \mathbf{X}_{k}, \mathbf{y} \in \mathbf{Y}_{k}}\left(\left[\mathbf{x}^{T}, \mathbf{y}^{T}\right]^{T}-\left[\mathbf{m}_{\mathbf{x}, k}, \mathbf{m}_{\mathbf{y}, k}\right]\right)\left(\left[\mathbf{x}^{T}, \mathbf{y}^{T}\right]^{T}-\left[\mathbf{m}_{\mathbf{x}, k}, \mathbf{m}_{\mathbf{y}, k}\right]\right)^{T}$ representing the within-class scatters, $\rho$ stands for the between-class margin to learn. $\lambda_{1}, \lambda_{2}$ and $\lambda_{3}$ are predefined hyper-parameters to control the weights of the objective terms.

In (3), the cross-view ordinal relationships are preserved by the model's constraints. In addition, the last term restricts the interdependencies between the $D$ th-directional projections and previously obtained $d$ projections as minimum as possible. (3) is a Quadratic-Programming (QP) function and can be solved using the off-the-shelf solving algorithms (e.g. [Lawrence 2000] [Radoslav 2006]).

\section{PROPOSED METHOD}

\subsection{Adaptive cross-view class center by moment-guided $l_{p}$-norm}

We can observe from (3) that the class center $\mathbf{m}_{k}$ (without loss of generality, we take the $k$ th class as example) is involved and plays critical role in restricting both the ordinal margins and and the data scatters. As defined in (3), $\mathbf{m}_{k}$ is derived from the Euclidean center of the $k$ th data class and can be detailed as

$$
\begin{aligned}
\left(\mathbf{m}_{k}\right)^{*} & =\arg \min _{\left\{\mathbf{m}_{k}\right\}} \sum_{\mathbf{x} \in \mathbf{X}_{k}, \mathbf{y} \in \mathbf{Y}_{k}}\left\|\left[\mathbf{m}_{\mathbf{x}, k}^{T}, \mathbf{m}_{\mathbf{y}, k}^{T}\right]^{T}-\left[\mathbf{x}^{T}, \mathbf{y}^{T}\right]^{T}\right\|_{2}^{2} \\
& =\arg \min _{\left\{\mathbf{m}_{k}\right\}} \sum_{\mathbf{x} \in \mathbf{X}_{k}, \mathbf{y} \in \mathbf{Y}_{k}}\left(\left[\mathbf{m}_{\mathbf{x}, k}^{T}, \mathbf{m}_{\mathbf{y}, k}^{T}\right]^{T}-\left[\mathbf{x}^{T}, \mathbf{y}^{T}\right]^{T}\right)^{T}\left(\left[\mathbf{m}_{\mathbf{x}, k}^{T}, \mathbf{m}_{\mathbf{y}, k}\right]^{T}-\left[\mathbf{x}^{T}, \mathbf{y}^{T}\right]^{T}\right) \\
& =\frac{1}{N_{k}} \sum_{\mathbf{x} \in \mathbf{X}_{k}, \mathbf{y} \in \mathbf{Y}_{k}}\left[\mathbf{x}^{T}, \mathbf{y}^{T}\right]^{T},
\end{aligned}
$$


where $N_{k}$ denotes the number of training samples from the $k$ th class.

Unfortunately, research [Kloft et al. 2009] [Nie et al. 2010] [Kloft et al. 2011] show that $l_{2}$-norm is sensitive to data outliers, hence they replaced $l_{2}$-norm with $l_{p}$-norm on the model parameters to control their model complexity and obtained accuracybetter recognition results. Motivated by the success of $l_{p}$-norm computation, in our previous work [Tian et al. 2018], we proposed to construct the class centers by using $l_{p}$-norm instead of $l_{2}$-norm and achieved promising recognition results on single view tasks. Unfortunately, the single-view class center deriving algorithm cannot be directly used to address cross-view data. Therefore, we definitely need to build a cross-view class center deriving algorithm which can alleviate the undesirable influence by data outliers. To this end, we formulate the $l_{p}$-norm derived cross-view class center as

$$
\left(\mathbf{m}_{k}\right)^{*}=\arg \min _{\mathbf{m}_{k}} \sum_{\left\{\mathbf{x}_{v} \in \mathbf{X}_{k v}\right\}_{v=1}^{V}}\left\|\left[\mathbf{x}_{1}^{T}, \cdots, \mathbf{x}_{V}^{T}\right]^{T}-\left[\mathbf{m}_{1 k}^{T}, \cdots, \mathbf{m}_{V k}^{T}\right]^{T}\right\|_{p}^{p}, p \in(0,2],
$$

in which $\mathbf{x}_{v}$ denotes one instance from the $k$ th class and $v$ th view representation $\mathbf{X}_{k v}$ of totally $V$ views. $\mathbf{m}_{k}=\left[\mathbf{m}_{1 k}^{T}, \cdots, \mathbf{m}_{V k}^{T}\right]^{T}$ denotes the entire class center for the $k$ th data class with $\mathbf{m}_{v k}$ being the $v$ th view center. Besides, $\|\mathbf{x}\|_{p}^{p}=\sum_{i=1}^{d}\left|\mathbf{x}_{i}\right|^{p}$ stands for the $p$-norm operator [Nie et al. 2010]. To solve $\mathbf{m}_{k}$ in (5), we construct a type of analytical solution for it, as follows

$$
\begin{aligned}
& \left(\mathbf{m}_{k}\right)^{*}=\arg \min _{\mathbf{m}_{k}} \sum_{\left\{\mathbf{x}_{v} \in \mathbf{X}_{k v}\right\}_{v=1}^{V}}\left\|\left[\mathbf{x}_{1}^{T}, \cdots, \mathbf{x}_{V}^{T}\right]^{T}-\left[\mathbf{m}_{1 k}^{T}, \cdots, \mathbf{m}_{V k}^{T}\right]^{T}\right\|_{p}^{p} \\
& =\arg \min _{\mathbf{m}_{k}} \sum_{\left\{\mathbf{x}_{v} \in \mathbf{X}_{k v}\right\}_{v=1}^{V}} \operatorname{tr}\left(\left(\left[\mathbf{x}_{1}^{T}, \cdots, \mathbf{x}_{V}^{T}\right]^{T}-\left[\mathbf{m}_{1 k}^{T}, \cdots, \mathbf{m}_{V k}^{T}\right]^{T}\right)^{T} D\left(\left[\mathbf{x}_{1}^{T}, \cdots, \mathbf{x}_{V}^{T}\right]^{T}-\left[\mathbf{m}_{1 k}^{T}, \cdots, \mathbf{m}_{V k}^{T}\right]^{T}\right)\right) \\
& =\arg \min _{\mathbf{m}_{k}} \sum_{\left\{\mathbf{x}_{v} \in \mathbf{X}_{k v}\right\}_{v=1}^{V}}\left(\left[\mathbf{x}_{1}^{T}, \cdots, \mathbf{x}_{V}^{T}\right]^{T}-\left[\mathbf{m}_{1 k}^{T}, \cdots, \mathbf{m}_{V k}^{T}\right]^{T}\right)^{T} D\left(\left[\mathbf{x}_{1}^{T}, \cdots, \mathbf{x}_{V}^{T}\right]^{T}-\left[\mathbf{m}_{1 k}^{T}, \cdots, \mathbf{m}_{V k}^{T}\right]^{T}\right) \\
& =\left(\sum_{\left\{\mathbf{x}_{v} \in \mathbf{X}_{k v}\right\}_{v=1}^{V}} D^{(t)}\right)^{-1}\left(\sum_{\left\{\mathbf{x}_{v} \in \mathbf{X}_{k v}\right\}_{v=1}^{V}} D^{(t)}\left[\mathbf{x}_{1}^{T}, \cdots, \mathbf{x}_{V}^{T}\right]^{T}\right),
\end{aligned}
$$

where $\operatorname{tr}(\cdot)$ denotes the trace operator, $D=\operatorname{diag}\left\{\left|\left[\mathbf{x}_{1}^{T}, \cdots, \mathbf{x}_{V}^{T}\right]^{T}-\left[\mathbf{m}_{1 k}^{T}, \cdots, \mathbf{m}_{V k}^{T}\right]^{T}\right|^{p-2}\right\}$ is a diagonal matrix and $D^{(t)}$ denotes the value at the $t$ th iteration.

We can see from (6) that, although the optimal multi-view centers of the data classes can be finally solved through an iterative optimization manner, the value of parameter $p$ needs to be assigned beforehand. Although we can set it by the cross-validation as in [Tian et al. 2018], thus manner is quite labor-intensive and even prohibitive in time-sensitive applications. To address this issue, it is preferable to efficiently set $p$ according to the distributions of training data. Along this line, we evaluate the value of $p$ in view of the statistical moments of training data. In this way, $p$ can be adaptively assigned without time-consuming grid search as in cross-validation.

Joint manner: Motivated by the work of [Kijko 1994], we propose to evaluate the parameter $p$ of (6) based on the first-, second- and fourth-order central moments of training data. Specifically, we denote $\left\{\widetilde{\mathbf{x}}_{k i}\right\}_{i=1}^{N}=\left\{\left[\mathbf{x}_{k i 1}^{T}, \cdots, \mathbf{x}_{k i V}^{T}\right]^{T}\right\}_{i=1}^{N}$ as the $V$-view concatenated representations of $N_{k}$ training instances from the the $k$ th class and calculate their residuals to corresponding class center $\mathbf{m}_{k}$ generated by (6), as follows

$$
\Delta \widetilde{x}_{k i}=\left\|\widetilde{\mathbf{x}}_{k i}-\mathbf{m}_{k}\right\|_{p}, i=1, \ldots, N
$$


Then, we respectively construct the first-, second- and fourth-order central moments of $\left\{\Delta \widetilde{x}_{k i}\right\}_{i=1}^{N}$ as

$$
\begin{gathered}
M_{1}=\frac{1}{N} \sum_{i=1}^{N} \Delta \widetilde{x}_{k i}, \\
M_{2}=\frac{1}{N-1} \sum_{i=1}^{N}\left(\Delta \widetilde{x}_{k i}-M_{1}\right)^{2}, \\
M_{4}=\frac{N^{2}-2 N-3}{(N-1)(N-2)(N-3)} \sum_{i=1}^{N}\left(\Delta \widetilde{x}_{k i}-M_{1}\right)^{4}-\frac{3(N-1)(2 N-3)}{N(N-2)(N-3)} M_{2}^{2} .
\end{gathered}
$$

Using the above generated statistical moments of training data, we then evaluate the value of $p$ according to the following equations

$$
\frac{M_{4}}{M_{2}^{2}}=\left\{\begin{array}{cc}
\frac{\Gamma(5 / p) \Gamma(1 / p)}{\Gamma(3 / p)^{2}}, & p \in(0,1) \\
\frac{6}{p}, & p \in[1,2]
\end{array}\right.
$$

where $\Gamma(\cdot)$ is the gamma function. According to (11), when $p \in(0,1)$, we can efficiently determine the value of $p$ by solving the gamma function using the secant method [Smith and Beattie 2015] or half-search [Bryson et al. 2007], or readily set it to $\frac{6 M_{2}^{2}}{M_{4}}$ if $p \in[1,2]$.

Separate manner: Actually, besides the joint manner of computing the moments of training data in (7)-(10), we can also estimate them in a separate manner for each dimension of the training data, one by one. To this end, we reformulate (7) as,

$$
\Delta \widetilde{x}_{k i d}=\widetilde{\mathbf{x}}_{k i d}-\mathbf{m}_{k d}, i=1, \ldots, N ; d=1, \ldots, D
$$

where $\widetilde{\mathbf{x}}_{k i d}$ and $\mathbf{m}_{k d}$ respectively denote the $d$ th-element of $\widetilde{\mathbf{x}}_{k i}$ and $\mathbf{m}_{k}$, whose dimensions are assumed to be $D$. Then, we can compute the first-, second- and fourth-order moments for each of the data dimensions in the way similar to (8)-(10). Next, we respectively average the second and fourth-order moments of all the dimensions as corresponding entire moments, and finally use them to calculate the parameter $p$ still based on (11).

Algorithm: We can see from (7) and (12) that, corresponding class center is involved in estimating its parameter $p$. In other words, in estimating one of $p$ and $\mathbf{m}_{k}$, the other is also involved. To this end, we construct an alternating algorithm to solve them. That is, we solve $\mathbf{m}_{k}$ through (6) by setting $p$ based on (11), and in turn recalculate $p$ based on (11) with updated $\mathbf{m}_{k}$. Then, we alternately repeat the above two steps until both of them converge. In this way, the centers of all the other classes can be obtained in the way. The complete optimization algorithm for calculating the adaptive moment-guided class centers for all the data classes is elaborated in Algorithm 1. We can see from Algorithm 1 that it is an iterative algorithm. To guarantee its convergence, we give Theorem 3.1:

THEOREM 3.1. Let $\left\{\boldsymbol{m}_{k}^{(t)}\right\}_{t=1}^{T}$ denote sequence of the generated kth class centers after $T$ iterations through Algorithm 1. Then, $f(t+1) \leqslant f(t), t=1, \ldots, T$. When $f(t+1)=f(t)$ holds, $\boldsymbol{m}_{k}^{*}=\boldsymbol{m}_{k}^{(t)}$ will be the saddle point of (6), meaning a (sub)optimal solution is obtained, whose proof is elaborated in the Appendix. 


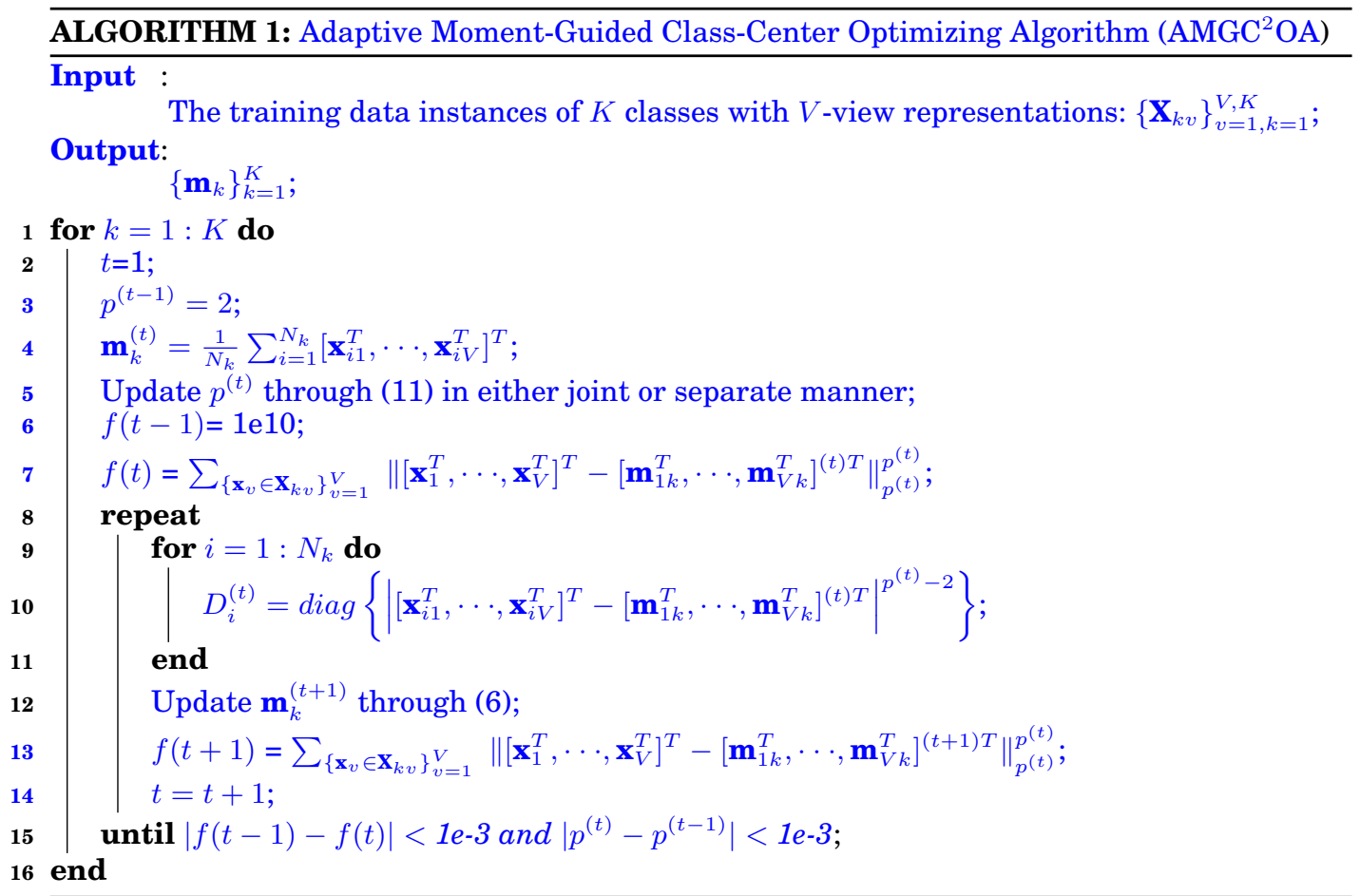

Through Algorithm 1, we will generate the cross-view class centers of the entire training data in an adaptive manner according to the data distribution moments. In addition, we can see from the definition interval $(0,2]$ of $p$ in $(6)$ that, the Euclidean center derived by $l_{2}$-norm is covered by the $l_{p}$-norm center. In other words, the $l_{p}$-norm center can better embody the statistical information of real data distributions.

\subsection{Preserving the data ordinal manifold structure}

It is not only important to generate outliers-insensitive cross-view class centers for correlation analysis, but also the spatial structures contained in training data instances. To this end, we adopt the manifold learning mechanism [He and Niyogi 2003] to model the cross-view spatial neighborhoods of the data. To preserve the ordinal characteristic of ordinal data concerned in this work, we build a type of ordinal manifold structure regularizer to preserve these data structures. To this end, we first define the ordinal spatial adjacency matrix $\mathbf{S}$ with its $(i, j)$ th element defined as,

$$
\mathbf{S}_{i j}=\exp \left(-\frac{\left(l_{i}-l_{j}\right)^{2}}{2 D i s}\right),
$$

where $l_{i}$ and $l_{j}$ respectively denote the class labels corresponding to the $i$ th and $j$ th instances. And, Dis stands for the averaged label difference of the training set which is defined as Dis $=\frac{1}{N^{2}} \sum_{i=1}^{N} \sum_{j=1}^{N}\left(l_{i}-l_{j}\right)^{2}$. As demonstrated in Fig. 1, the similarity between faces aged 4 and 5 is greater than that between 5 and 7 , so the former should be assigned with higher weight than the latter. Then, by defining adjacency matrices $\left\{\mathbf{S}^{v}\right\}_{v=1}^{V}$ in the same way for each of the $V$ views, we can achieve the goal of preserving 


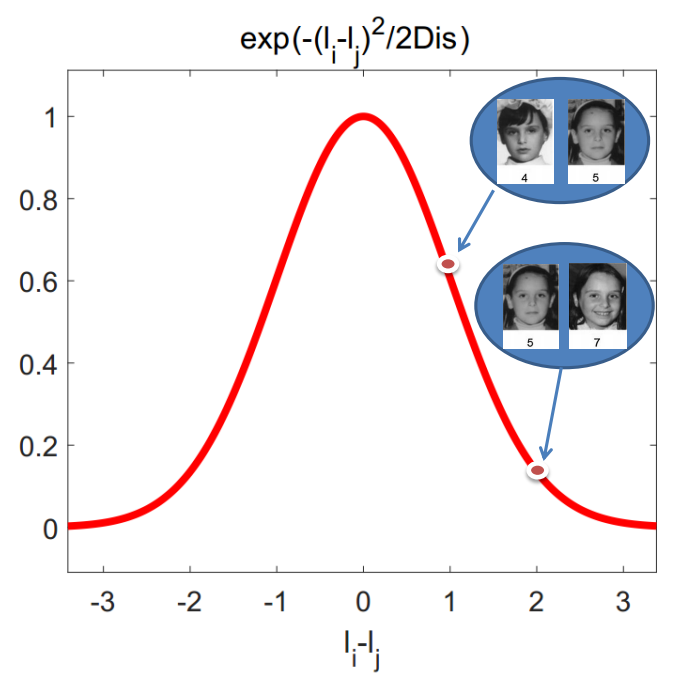

Fig. 1. Illustration of adjacency relationships between neighboring human age labels. $l_{i}$ and $l_{j}$ herein respectively denote the class labels of the $i$ th and $j$ th instances.

the cross-view ordinal structures of training data instances by

$$
\begin{aligned}
& \min _{\left\{\mathbf{w}_{v}\right\}_{v=1}^{V}} \sum_{v=1}^{V} \sum_{i=1}^{N} \sum_{j=1}^{N} \frac{1}{2}\left\|\mathbf{w}_{v}^{T} \mathbf{x}_{i v}-\mathbf{w}_{v}^{T} \mathbf{x}_{j v}\right\|^{2} \mathbf{S}_{i j}^{v} \\
& =\sum_{v=1}^{V}\left(\sum_{i}^{N} \mathbf{w}_{v}^{T} \mathbf{x}_{i v} \mathbf{D}_{i i}^{v} \mathbf{x}_{i v}^{T} \mathbf{w}_{v}-\sum_{i=1}^{N} \sum_{j=1}^{N} \mathbf{w}_{v}^{T} \mathbf{x}_{i v} \mathbf{S}_{i j}^{v} \mathbf{x}_{j v}^{T} \mathbf{w}_{v}\right) \\
& =\sum_{v=1}^{V} \mathbf{w}_{v}^{T} \mathbf{X}_{v} \mathbf{L}^{v} \mathbf{X}_{v}^{T} \mathbf{w}_{v}
\end{aligned}
$$

where $\mathbf{X}^{v}$ stands for the $v$ th view data set, $\mathbf{L}^{v}=\mathbf{D}^{v}-\mathbf{S}^{v}$ denote the Laplacian matrices with $\mathbf{D}^{v}$ as diagonal matrices whose elements $\mathbf{D}_{i i}^{v}=\sum_{j=1}^{N} \mathbf{S}_{i j}^{v}$. Related works [Chu and Keerthi 2007], [Tian et al. 2016b], [Tian et al. 2016a] show that taking into account such ordinal structures will boost subsequent ordinal regression accuracy. Moreover, preserving the spatial ordinal structure of data can help mitigate the efforts of outliers to data ground-truth distributions.

\subsection{Moment-guided cross-view discriminative ordinal correlation regression}

To evaluate the efficacy of the moment-guided class center algorithm and ordinal structure preserving scheme, without loss of generality, we remodel (3) to multi-view scenarios by 1) replacing the class centers with the proposed moment-guided centers, and 2) incorporating the ordinal manifold structure regularizer in the objective. More specifically, we replace its class centers by (6) and add (14) as a regularizer in (3) and consequently obtain a robust manifold ordinal discriminative correlation regression 
(rmODCR, for short), formulated as,

$$
\begin{aligned}
& \min _{\left\{\mathbf{w}_{d}=\left[\mathbf{w}_{d 1}^{T}, \ldots, \mathbf{w}_{d V}^{T}\right]^{T}, \rho\right\}} \frac{1}{V(V-1)} \sum_{m=1}^{V} \sum_{n=m}^{V}\left\|\mathbf{w}_{d m}^{T} \mathbf{X}_{m}-\mathbf{w}_{d n}^{T} \mathbf{X}_{n}\right\|_{2}^{2}+\lambda_{1} \mathbf{w}_{d}^{T} \mathbf{S}_{w} \mathbf{w}_{d}-\lambda_{2} \rho \\
& \quad+\lambda_{3} \sum_{h=1}^{d-1}\left(\mathbf{w}_{h}^{T} \mathbf{w}_{d}\right)^{2}+\frac{\lambda_{4}}{V} \sum_{h=1}^{V} \mathbf{w}_{d h}^{T} \mathbf{X}_{h} \mathbf{L}^{h} \mathbf{X}_{h}^{T} \mathbf{w}_{d h} \\
& \text { s.t. } \quad \mathbf{w}_{d}^{T}\left(\mathbf{m}_{k+1}-\mathbf{m}_{k}\right) \geq \rho, k=1, \ldots, K-1
\end{aligned}
$$

where $\mathbf{m}_{k}$ and $\mathbf{m}_{k+1}$ are the generated class centers through Algorithm 1. We equivalently transform (15) as

$$
\begin{aligned}
& \min _{\left\{\mathbf{w}_{d}, \rho\right\}} \mathbf{w}_{d}^{T} \mathbf{M}_{d} \mathbf{w}_{d}-\lambda_{2} \rho \\
& \text { s.t. } \quad \mathbf{w}_{d}^{T}\left(\mathbf{m}_{k+1}-\mathbf{m}_{k}\right) \geq \rho, k=1, \ldots, K-1
\end{aligned}
$$

in which,

$$
\begin{aligned}
\mathbf{M}_{d}= & \frac{1}{V(V-1)} \sum_{m=1}^{V} \sum_{n=m}^{V}\left(\left(\mathbf{E}_{m}+\mathbf{E}_{n}\right)\left[\mathbf{X}_{m}^{T},-\mathbf{X}_{n}^{T}\right]^{T}\left[\mathbf{X}_{m}^{T},-\mathbf{X}_{n}^{T}\right]\left(\mathbf{E}_{m}+\mathbf{E}_{n}\right)^{T}\right)+\lambda_{1} \mathbf{S}_{w} \\
& +\lambda_{3} \sum_{h=1}^{d-1} \mathbf{w}_{h} \mathbf{w}_{h}^{T}+\frac{\lambda_{4}}{V} \operatorname{diag}\left\{\mathbf{X}_{1} \mathbf{L}^{1} \mathbf{X}_{1}^{T}, \ldots, \mathbf{X}_{V} \mathbf{L}^{V} \mathbf{X}_{V}^{T}\right\}
\end{aligned}
$$

with $\mathbf{E}_{m}$ being a matrix composed by the $\left(\sum_{i=1}^{m-1} d_{i}+1\right)$ th to $\left(\sum_{i=1}^{m} d_{i}\right)$ th columns of a $\left(\sum_{i=1}^{V}\right)$-order identity matrix. To make the optimization more efficient, we alternatively solve its dual problem, which is formulated as

$$
\begin{aligned}
& \min _{\{\boldsymbol{\alpha}\}} \sum_{k=1}^{K-1} \boldsymbol{\alpha}_{k}\left(\mathbf{m}_{k+1}-\mathbf{m}_{k}\right)^{T} \mathbf{M}_{d}^{-1}\left(\mathbf{m}_{k+1}-\mathbf{m}_{k}\right) \boldsymbol{\alpha}_{k} \\
& \text { s.t. } \quad \boldsymbol{\alpha}_{k} \geq 0, \sum_{k=1}^{K-1} \boldsymbol{\alpha}_{k}=\lambda_{2},
\end{aligned}
$$

where $\alpha \in \mathbb{R}^{K-1}$ is the dual variable. Obviously, (18) is QP with respect to $\alpha$ and can be solved using the QP optimization toolboxes [Lawrence 2000] [Radoslav 2006]. After $\boldsymbol{\alpha}$ is solved, we can recover $\mathbf{w}_{d}=\frac{1}{2} \mathbf{M}_{d}^{-1} \sum_{k=1}^{K-1} \boldsymbol{\alpha}_{k}\left(\mathbf{m}_{k+1}-\mathbf{m}_{k}\right)$. In a similar way, we can consequently generate $d$ regression projection directions $\mathbf{W}=\left[\mathbf{w}_{1}, \ldots, \mathbf{w}_{d}\right]$ and use them to conduct cross-view regression on ordinal test data.

\section{EXPERIMENTS}

\subsection{Setup}

To comprehensively evaluate the proposed model and algorithm, we conducted crossview refression experiments on five typical ordinal databases, i.e., MFD [Breukelen et al. 1998], USPS, UMIST [Timo et al. 2006], CACD [Chen et al. 2015] and YaleB [Kuang-Chih et al. 2005]. We compared the proposed methods with several related works, they are RSCCA [Shi et al. 2017], MVDA [Kan et al. 2015], OR-DisCCA [Zhou and Chen 2014], sepOrCCA [Kawashima et al. 2013], DeepCCA [Andrew et al. 2013]. To evaluate the effectiveness of the joint and separate manner of the proposed Algorithm 1 , we respectively name the proposed methods with the parameter $p$ estimated 
through joint manner, separate manner and cross-validation manner (interval [0.1, $0.2, \ldots, 2])$ as "rmODCR-joint", "rmODCR-separate" and "rmODCR-CV". To evaluate the proposed models in distinguishing nonlinear patterns, we also extended rmODCR by taking it as the loss objective of deep convolutional models and fine-tuning the model parameters. To distinguish it from rmODCR, we call it "Deep-rmODCR". The experiments were conducted on a platform with i9-9900K-4.7GHz-8Core CPU, 32GB memory and GeForce-GTX-1060 GPU. As reported in previous works, we also adopted the MAE (Mean Absolute Errors) as ordinal estimation performance measure. For the other hyper-parameters of the proposed methods, both $\lambda_{1}$ and $\lambda_{3}$ were cross-validated in the range of [1e-1, 1e $0,1 \mathrm{e} 1,1 \mathrm{e} 2,1 \mathrm{e} 3]$, the value of $\lambda_{4}$ was grid-searched in the range of $[1 \mathrm{e}-6,1 \mathrm{e}-5,1 \mathrm{e}-4,1 \mathrm{e}-3,1 \mathrm{e}-2]$, and $\lambda_{2}$ was fixed to 10 throughout the experiments. As for the parameters of the compared methods, they were also tuned through crossvalidation by referring to individual literature. Unless otherwise specified, all the reported results are a average of ten runs with random data partitions (the training and test sets did not intersect).

\subsection{Experiment on MFD}

MFD is one famous multi-view benchmark database represented by six feature views and is composed of 0-9 handwritten digits, each with 200 samples. At first, we each time chose two from the fix views to perform evaluations and generated totally fifteen combinations. Then, we performed evaluations by using all the feature views simultaneously. For the experiments, we took fifty percentage of samples for model training while the rest for test. For final regression decision, we assign a class label for a test instance to whose center the distance is the nearest. To evaluate the performance of the models against sample noises (outliers), we randomly corrupted certain ratios of samples with noises. The results are respectively reported in Fig. 2 and Fig. 3.

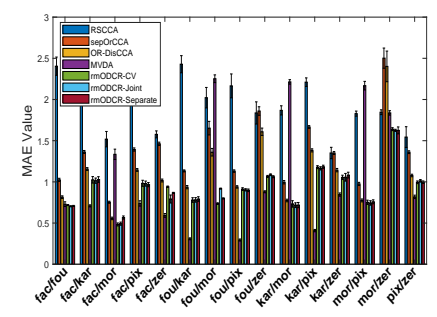

(a) Noise percentage: $0 \%$

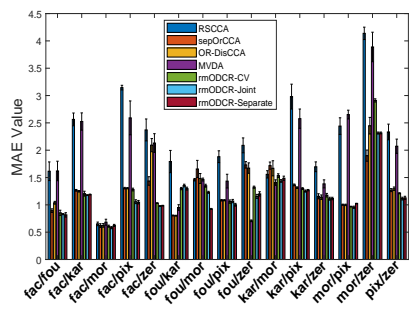

(b) Noise percentage: $10 \%$

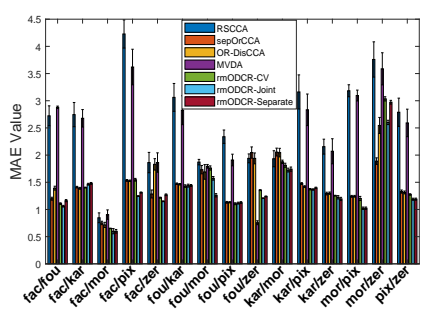

(c) Noise percentage: $20 \%$

Fig. 2. Estimation results on MFD with two-view combinations.

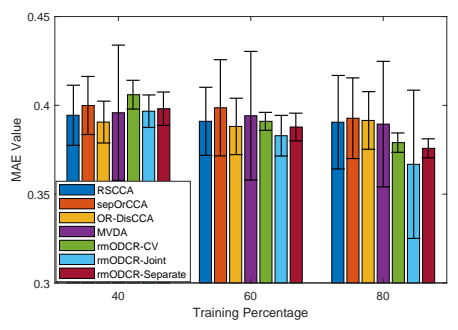

Fig. 3. Results on MFD evaluated on all six feature views. 
We can observe the following findings from the results. First, when clean training data was applied for model training, the estimation accuracy of MVDA is the highest among all the compared methods. However, when data noises are involved from $10 \%$ to $20 \%$, its performance becomes worse than the proposed rmODCR methods. It shows that although the cross-view semantic correlation is definitely preserved in MVDA and can generates promising results, it will deteriorate if the training data is corrupted with data noises since it estimates cross-view correlations and scatter of training data with data centers which are computed through the noise-sensitive Euclidean norm. By contrast, with increased ratio of instance outliers in training data, the proposed rmODCR models (i.e. rmODCR-CV, rmODCR-Joint and rmODCR-Separate) become to enjoy increasing performance superiority, due to that they are built with data centers that are derived by noise-insensitive $l_{p}$-norm. Second, compared with rmODCR-CV, the rmODCR-Joint and rmODCR-Separate methods generated comparative or even better estimation results. It shows that compared to calculating the class centers through empirical cross-validation manner, estimating them adaptively according to data distributions (i.e. data moments) in an data-driven manner is preferable. Last but not least,, in most of the comparative results, rmODCR-Joint is better than rmODCRSeparate. It states that estimating the data feature moments in joint manner is superior to estimating each of the feature dimensions separately, in both two-view and multi-view scenarios.

\subsection{Experiments on USPS and UMIST}

USPS and UMIST are two widely used data sets to estimate ordinal models. For USPS, it is also a handwritten digit database containing 11000 digital images of $0-9$, each with 1100 instances. As for UMIST, it is a head-pose estimation data set composed of about 564 face images of 20 people, angled from frontal to profile. For the purpose of crossview regression, we respectively drawn gray pixels and HoG parameters [Kobayashi and Otsu 2008] from normalized images and extracted 95\% PCA components from them as two feature views from the two databases. The images of UMIST are grouped into 6 categories by the face skew angles. For Deep-rmODCR, we respectively took the pretrained VGG16 [Simonyan and Zisserman 2014] and AlexNet [Alex et al. 2012] to generate two-view deep representations by replacing their loss objectives with that of rmODCR and fine-tuning them on the training image data.

4.3.1. Comparison results. With above settings, the ordinal digital and head pose estimation results are shown in Fig. 4 and Fig. 5.

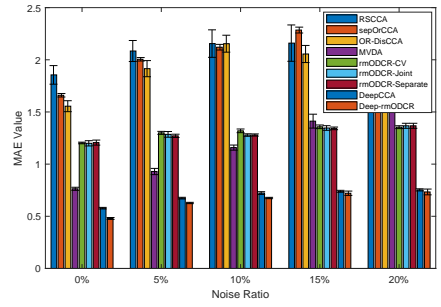

(a) Training percentage: $10 \%$

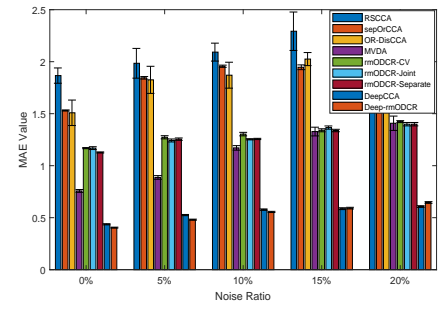

(b) Training percentage:30\%

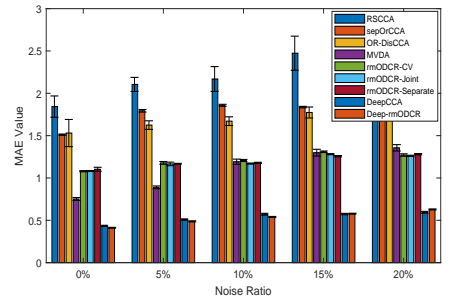

(c) Training percentage:50\%

Fig. 4. Digital estimation results on USPS with varying ratio of training data. 


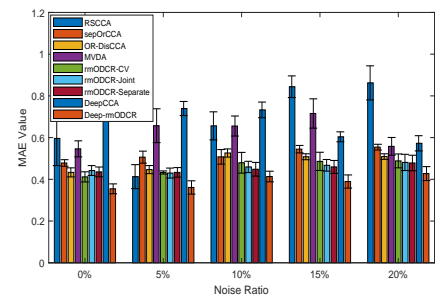

(a) Training percentage: $40 \%$

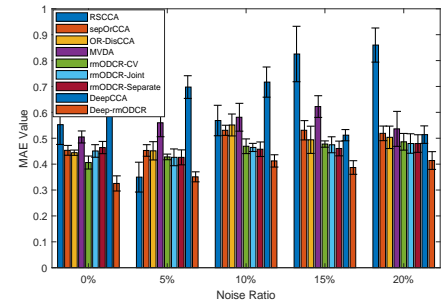

(b) Training percentage:60\%

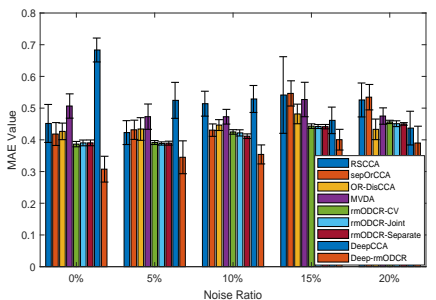

(c) Training percentage: $80 \%$

Fig. 5. Digital estimation results on UMIST with varying ratio of training data.

The following findings can be observed from the results in Fig. 4 and Fig. 5. First, within each of the subfigures, with increased percentage of noises, the estimation accuracies of all the methods become to deteriorate. It once again claims that for model training, corrupted instance outliers (noises) definitely smear the model performance. Second, with increased percentage of training samples, the estimation accuracies of all the compared methods are accordingly ascend. This observation corroborates that applying adequately more data for model training can definitely boost its generalization performance. Third, with increased percentage of training data noise, the performance superiority of rmODCR and its variants to others is becoming more significant. Moreover, the estimation accuracy of rmODCR-Joint is better than rmODCR-CV and rmODCR-Separate. These observations corroborate once again that calculating the class centers of cross-view ordinal regressors by jointly taking into account the moments information of all the data feature dimensions is preferable to modelling each of them separately. More importantly, it claims the superiority of calculating the data centers through moment-guided $l_{p}$-norm where the parameter $p$ is estimated according to the moments of the data distributions. Last but not least, the deep model DeepCCA yielded worse results than other ordinal shallow models. It is largely due to the ordinal characteristic of data is not preserved in DeepCCA. By contrast, the proposed deep model, i.e. Deep-rmODCR, achieved the best results in all comparison cases. It witnesses the effectiveness of our modeling schemes.

4.3.2. Parameter analysis. We also experimentally evaluated the efficacy of the withinclass scatter preserving subobjective and data structure preserving subobjective of (15), which respectively correspond to the $\lambda_{1}$ and $\lambda_{4}$ terms of (15). Without loss of generality, we show the results on USPS and UMIST in Fig. 6 and Fig. 7. We can observe two-fold findings. First, compared to the estimation performance of the regressor trained by clean data, the performance of the regressor by noise-corrupted data is worse. It coincides with aforementioned findings that data noise smear regressors' performance. Second, on both USPS and UMIST, the performance rule along the $\lambda_{1}$ direction does not coincide with that along the $\lambda_{4}$ direction. It claims that the scatter components are not contained by the data structure preserving term, and vice versa. As a result, both of them should be incorporated in the objective simultaneously. That is, besides the data scatter components, the data ordinal spatial structures should also be preserved.

\subsection{Experiments on CACD and YaleB}

To evaluate the efficacy of the proposed methodology in handling more challenging large-scale ordinal data sets, we also experimented on CACD and YaleB. Specifically, 


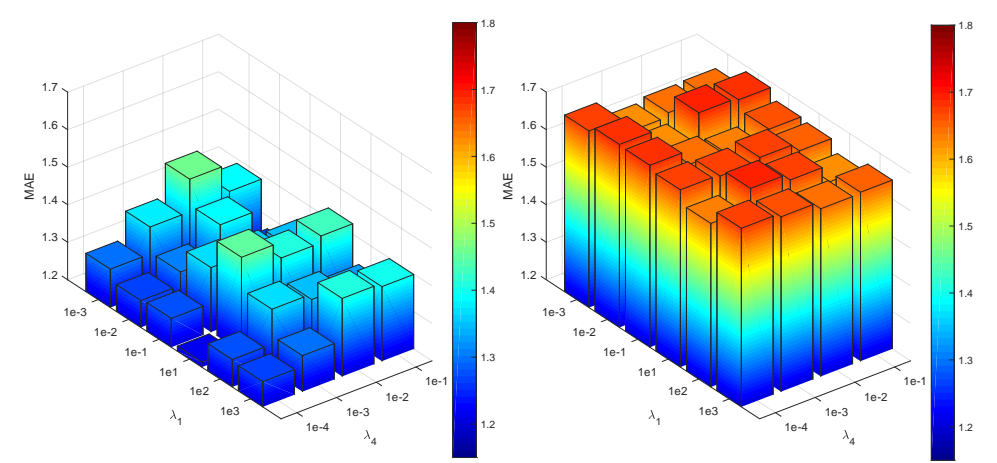

Fig. 6. Estimation results on USPS with varying $\lambda_{1}$ and $\lambda_{4}$ combinations, without (left) and with $20 \%$ (right) noise corruptions.

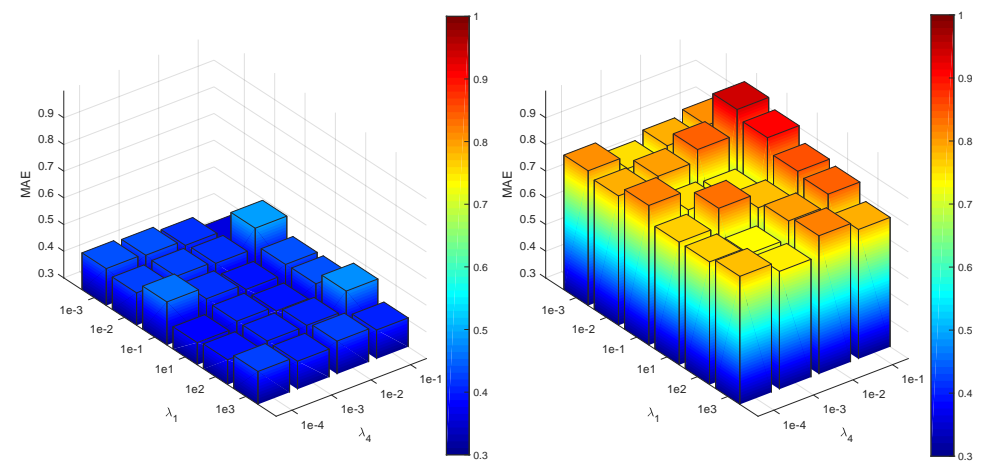

Fig. 7. Estimation results on UMIST with varying $\lambda_{1}$ and $\lambda_{4}$ combinations, without (left) and with $20 \%$ (right) noise corruptions.

the CACD dataset is composed of over 160000 face images from 2000 celebrities, aged between 16 and 62 years. The facial age annotations of the images are inaccurate or even wrong. In other words, the CACD dataset is naturally corrupted by instance outliers. As for YaleB, it is composed of 16129 facial images in 64 lighting angles of 9 head poses took from 28 people. For the purpose of multi-view regression, we took images from the same head pose as a representation view and generated totally 9 views. From CACD, we extracted LBP and HoG features and kept their 95\% components as final two views and just HoG features from YaleB with keeping the $95 \%$ components as view representations from each of the nine poses, for all the compared shallow models. On CACD, we also evaluated Deep-rmODCR by adopting the pre-tranined VGG16 and AlexNet networks and fine-tuning them with the objective function of rmODCR.

4.4.1. Comparison results. With the above settings, we conducted comparison experiments and show the results in Fig. 8 and Fig. 9. We can observe the following findings. First, with increased training data on both CACD and YaleB, estimation errors of al1 the methods are descending. It shows that training the estimators with more data can improve their performance on large-scale challenging tasks. Second, the estimations results of rmODCR-CV, rmODCR-Joint and rmODCR-Separate are comparable, and more importantly, rmODCR-Joint and rmODCR-Separate surpass rmODCR-CV in most cases. It once again shows that estimating the data centers adaptively accord- 
ing to the moments of data is preferable than manual cross-validation manner. Third, the architecture deepened counterpart of rmODCR, i.e. Deep-rmODCR, generated the best estimation results (the lowest MAEs) among all the methods, it verifies the solidness of our architecture deepening scheme.

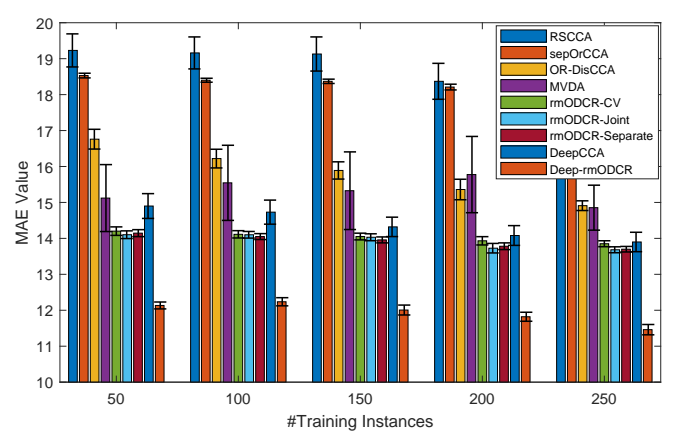

Fig. 8. Face estimation on CACD represented with two views.

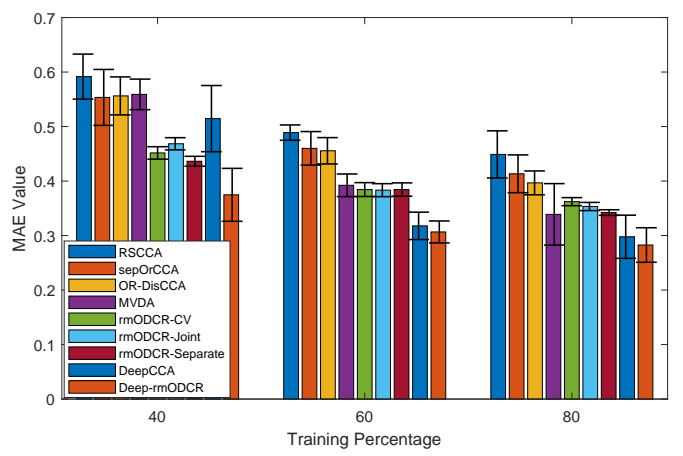

Fig. 9. Face estimation on YaleB represented with nine views.

4.4.2. Convergence analysis. On the CACD dataset, we also evaluated the convergence efficiency of the proposed Algorithm 1 with respect to the parameter $p$ and the objective value. The results are demonstrated in Fig. 10 and Fig. 11. We can observe from them that both the parameter $p$ and objective value of Algorithm 1 efficiently converge within about five iterations.

4.4.3. Time complexity comparison. With the same setting, we also evaluated the time complexity of the methods on the CACD dataset. The results are shown in Table I, where the boldface results are the lowest with statistical significance (t-test with $\mathrm{p}=0.05$ ). We can observe the following findings. First, with increased number of training instances, the time cost of all the methods ascend, among which the time cost of sepOrCCA is significantly the lowest. It is because only one projection direction is involved in sepOrCCA, while multiple directions are solved in the other methods. Second, the time complexities of rmODCR-Joint and rmODCR-Separate are comparable to the other multi-direction methods; however, the time cost of rmODCR-CV is about 


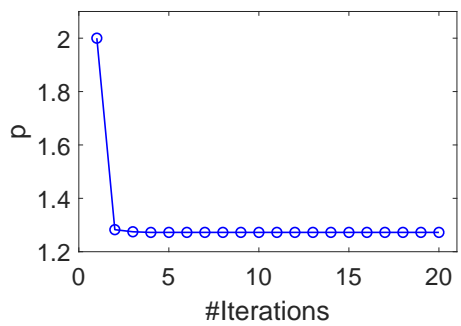

(a) In joint manner

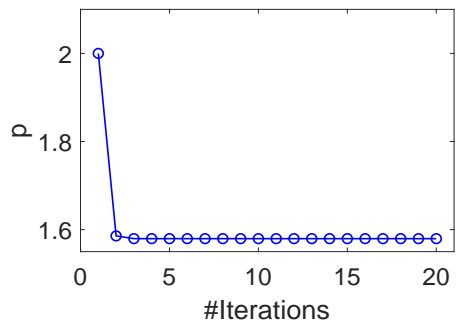

(b) In separate manner

Fig. 10. Convergence rule on $p$ of Algorithm 1 in joint and separate manners.

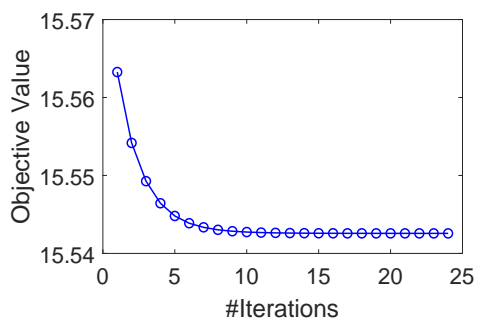

(a) In joint manner

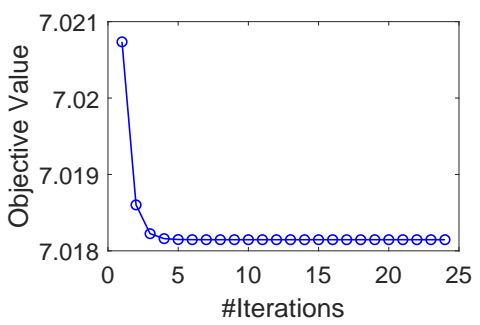

(b) In separate manner

Fig. 11. Convergence rule on the objective of Algorithm 1 in joint and separate manners.

an order of magnitude longer than that of rmODCR-Joint and rmODCR-Separate. It verifies that estimating data centers according to their moments is more efficient than cross-validation manner. Third, our architecture-deepened model (i.e. Deep-rmODCR) also costed less time than the DeepCCA model, which confirms the relatively lower time complexity of our methodology.

Table I. Time complexity comparison on CACD (in seconds).

\begin{tabular}{|c|c|c|c|c|c|c|c|c|c|}
\hline \#Training Instances & RSCCA & sepOrCCA & OR-DisCCA & MVDA & rmODCR-CV & rmODCR-Joint & rmODCR-Separate & DeepCCA & Deep-rmODCR \\
\hline 50 & 1.18 & 0.03 & 0.75 & 2.41 & 64.43 & 2.53 & 2.24 & 24.31 & 8.31 \\
\hline 150 & 8.11 & 0.05 & 0.76 & 13.23 & 70.29 & 2.85 & 2.43 & 103.54 & 56.05 \\
\hline 250 & 21.65 & 0.10 & 0.78 & 30.31 & 74.32 & 3.79 & 3.20 & 221.86 & 149.72 \\
\hline
\end{tabular}

\section{CONCLUSION}

In this paper, we constructed a robust manifold-preserved ordinal multi-view discriminative correlation regression model, coined as rmODCR, whose noise-insensitiveness is achieved by replacing the traditional $l_{2}$-norm data centers with $l_{p}$-norm centers where $p$ is efficiently estimated according to the moments of the data distributions in both joint and separate manners, as well as by incorporating the manifold distribution information of the data in the objective optimization. We also provided theoretical convergence guarantee for the proposed algorithm. In addition, we further extended rmODCR with deep convolutional architectures. Through extensive experiments, we evaluated the efficacy and superiority of the proposed methods. In future, we will consider to explore the cross-moment correlations among the data views and incorporate it to boost the performance. 


\section{ACKNOWLEDGMENT}

This work was partially supported by the National Natural Science Foundation of China under grant 61702273, the Natural Science Foundation of Jiangsu Province under grant BK20170956, the Natural Science Foundation of the Jiangsu Higher Education Institutions of China under grant 17KJB520022, the Fundamental Research Funds for the Central Universities No. NJ2019010, the Project Funded by the Priority Academic Program Development of Jiangsu Higher Education Institutions, the Priority Academic Program Development of Jiangsu Higher Education Institutions (PAPD) fund, and the Startup Foundation for Talents of Nanjing University of Information Science and Technology.

\section{APPENDIX}

LEMmA A.1. Let $\varphi(\lambda)=\lambda-a \lambda^{a}$ with $a \in(0,1)$. Then for any $\lambda>0$, we have $\varphi(\lambda) \leqslant 1-a$ and $\lambda=1$ is the unique maximizer.

Proof: By taking the derivative of $\varphi(\lambda)$ with respective to $\lambda$ and set it to zero, we get

$$
\varphi^{\prime}(\lambda)=1-t^{\frac{1}{a}-1}=0,
$$

which has the unique solution $\lambda=1$, given that $a \in(0,1)$ and $\lambda>0$.

LEMMA A.2. Let $\left\{\boldsymbol{x}_{i v}\right\}_{i=1, v=1}^{N_{k}, V}$ denote $V$-view representations of $N_{k}$ training samples from the kth class, $\left\{\boldsymbol{m}_{k}^{(t)}\right\}_{t=1}^{T}$ denote the sequence of the generated kth class centers through Algorithm 1, and let $f(t)$ denote the objective value of (6) after t iterations. Then, it holds that

$$
\begin{aligned}
& N_{k} f(t+1)-\frac{p}{2} \sum_{i=1}^{N_{k}}\left(\left[\boldsymbol{x}_{i 1}^{T}, \cdots, \boldsymbol{x}_{i V}^{T}\right]^{T}-\left[\boldsymbol{m}_{1 k}^{T}, \cdots, \boldsymbol{m}_{V k}^{T}\right]^{(t+1) T}\right)^{T} D_{i}^{(t)}\left(\left[\boldsymbol{x}_{i 1}^{T}, \cdots, \boldsymbol{x}_{i V}^{T}\right]^{T}-\left[\boldsymbol{m}_{1 k}^{T}, \cdots, \boldsymbol{m}_{V k}^{T}\right]^{(t+1) T}\right) \\
& \leqslant\left(1-\frac{p}{2}\right) N_{k} f(t) .
\end{aligned}
$$

Proof: Let $a=\frac{p}{2} \in(0,1)$ with $p \in(0,2)$, and $\lambda=\frac{\left|\left[\mathbf{x}_{i 1}^{T}, \cdots, \mathbf{x}_{i V}^{T}\right]_{j}^{T}-\left[\mathbf{m}_{1 k}^{T}, \cdots, \mathbf{m}_{V k}^{T}\right]_{j}^{(t+1) T}\right|^{p}}{\left|\left[\mathbf{x}_{i 1}^{T}, \cdots, \mathbf{x}_{i V}^{T}\right]_{j}^{T}-\left[\mathbf{m}_{1 k}^{T}, \cdots, \mathbf{m}_{V k}^{T}\right]_{j}^{(t) T}\right|^{p}}$, $j=1, \ldots, d$, where $[\cdot]_{j}$ indicates the $j$ th element of a $d$-dimensional vector. Then, it holds that

$$
\begin{aligned}
& \frac{\left|\left[\mathbf{x}_{i 1}^{T}, \cdots, \mathbf{x}_{i V}^{T}\right]_{j}^{T}-\left[\mathbf{m}_{1 k}^{T}, \cdots, \mathbf{m}_{V k}^{T}\right]_{j}^{(t+1) T}\right|^{p}}{\left|\left[\mathbf{x}_{i 1}^{T}, \cdots, \mathbf{x}_{i V}^{T}\right]_{j}^{T}-\left[\mathbf{m}_{1 k}^{T}, \cdots, \mathbf{m}_{V k}^{T}\right]_{j}^{(t) T}\right|^{p}}-\frac{p}{2} \frac{\left|\left[\mathbf{x}_{i 1}^{T}, \cdots, \mathbf{x}_{i V}^{T}\right]_{j}^{T}-\left[\mathbf{m}_{1 k}^{T}, \cdots, \mathbf{m}_{V k}^{T}\right]_{j}^{(t+1) T}\right|^{2}}{\left|\left[\mathbf{x}_{i 1}^{T}, \cdots, \mathbf{x}_{i V}^{T}\right]_{j}^{T}-\left[\mathbf{m}_{1 k}^{T}, \cdots, \mathbf{m}_{V k}^{T}\right]_{j}^{(t) T}\right|^{2}} \\
& \leqslant 1-\frac{p}{2},
\end{aligned}
$$

which is equivalent to

$$
\begin{aligned}
& \left|\left[\mathbf{x}_{i 1}^{T}, \cdots, \mathbf{x}_{i V}^{T}\right]_{j}^{T}-\left[\mathbf{m}_{1 k}^{T}, \cdots, \mathbf{m}_{V k}^{T}\right]_{j}^{(t+1) T}\right|^{p}-\frac{p}{2} \frac{\left|\left[\mathbf{x}_{i 1}^{T}, \cdots, \mathbf{x}_{i V}^{T}\right]_{j}^{T}-\left[\mathbf{m}_{1 k}^{T}, \cdots, \mathbf{m}_{V k}^{T}\right]_{j}^{(t+1) T}\right|^{2}}{\left|\left[\mathbf{x}_{i 1}^{T}, \cdots, \mathbf{x}_{i V}^{T}\right]_{j}^{T}-\left[\mathbf{m}_{1 k}^{T}, \cdots, \mathbf{m}_{V k}^{T}\right]_{j}^{(t) T}\right|^{2-p}} \\
& \leqslant\left(1-\frac{p}{2}\right)\left|\left[\mathbf{x}_{i 1}^{T}, \cdots, \mathbf{x}_{i V}^{T}\right]_{j}^{T}-\left[\mathbf{m}_{1 k}^{T}, \cdots, \mathbf{m}_{V k}^{T}\right]_{j}^{(t) T}\right|^{p} .
\end{aligned}
$$


By accumulating on $j=1, \ldots, d$ and $i=1, \ldots, N_{k}$ respectively for both sides of (22), we can obtain

$$
\begin{aligned}
& \sum_{i=1}^{N_{k}}\left\|\left[\mathbf{x}_{1}^{T}, \cdots, \mathbf{x}_{V}^{T}\right]^{T}-\left[\mathbf{m}_{1 k}^{T}, \cdots, \mathbf{m}_{V k}^{T}\right]^{(t+1) T}\right\|_{p}^{p} \\
& -\frac{p}{2} \sum_{i=1}^{N_{k}}\left(\left[\mathbf{x}_{1}^{T}, \cdots, \mathbf{x}_{V}^{T}\right]^{T}-\left[\mathbf{m}_{1 k}^{T}, \cdots, \mathbf{m}_{V k}^{T}\right]^{(t+1) T}\right)^{T} D_{i}^{(t)}\left(\left[\mathbf{x}_{1}^{T}, \cdots, \mathbf{x}_{V}^{T}\right]^{T}-\left[\mathbf{m}_{1 k}^{T}, \cdots, \mathbf{m}_{V k}^{T}\right]^{(t+1) T}\right) \\
& \leqslant\left(1-\frac{p}{2}\right) \sum_{i=1}^{N_{k}}\left\|\left[\mathbf{x}_{1}^{T}, \cdots, \mathbf{x}_{V}^{T}\right]^{T}-\left[\mathbf{m}_{1 k}^{T}, \cdots, \mathbf{m}_{V k}^{T}\right]^{(t) T}\right\|_{p}^{p}
\end{aligned}
$$

That is,

$$
\begin{aligned}
& N_{k} f(t+1)-\frac{p}{2} \sum_{i=1}^{N_{k}}\left(\left[\mathbf{x}_{1}^{T}, \cdots, \mathbf{x}_{V}^{T}\right]^{T}-\left[\mathbf{m}_{1 k}^{T}, \cdots, \mathbf{m}_{V k}^{T}\right]^{(t+1) T}\right)^{T} D_{i}^{(t)}\left(\left[\mathbf{x}_{1}^{T}, \cdots, \mathbf{x}_{V}^{T}\right]^{T}-\left[\mathbf{m}_{1 k}^{T}, \cdots, \mathbf{m}_{V k}^{T}\right]^{(t+1) T}\right) \\
& \leqslant\left(1-\frac{p}{2}\right) N_{k} f(t) .
\end{aligned}
$$

According to Lemma A.1, the equation case of (24) holds if and only if $\lambda=\frac{\left|\left[\mathbf{x}_{i 1}^{T}, \cdots, \mathbf{x}_{i V}^{T}\right]_{j}^{T}-\left[\mathbf{m}_{1 k}^{T}, \cdots, \mathbf{m}_{V k}^{T}\right]_{j}^{(t+1) T}\right|^{p}}{\left|\left[\mathbf{x}_{i 1}^{T}, \cdots, \mathbf{x}_{i V}^{T}\right]_{j}^{T}-\left[\mathbf{m}_{1 k}^{T}, \cdots, \mathbf{m}_{V k}^{T}\right]_{j}^{(t) T}\right|^{p}}, j=1, \ldots, d$, which means that $\left(m_{k}\right)^{(t+1)}=\left(m_{k}\right)^{(t)}$.

\section{Proof of Theorem 3.1:}

Since $\mathbf{m}_{k}^{(t+1)}$ denotes the solution of Algorithm 1 after $t$ iterations. Then, it holds that $\mathbf{m}_{k}^{(t+1)}=\arg \min _{\mathbf{m}_{k}} \sum_{i=1}^{N_{k}}\left(\left[\mathbf{x}_{1}^{T}, \cdots, \mathbf{x}_{V}^{T}\right]^{T}-\left[\mathbf{m}_{1 k}^{T}, \cdots, \mathbf{m}_{V k}^{T}\right]^{(t) T}\right)^{T} D_{i}^{(t)}\left(\left[\mathbf{x}_{1}^{T}, \cdots, \mathbf{x}_{V}^{T}\right]^{T}-\left[\mathbf{m}_{1 k}^{T}, \cdots, \mathbf{m}_{V k}^{T}\right]^{(t) T}\right)$,

As a result, we have

$$
\begin{aligned}
& \sum_{i=1}^{N_{k}}\left(\left[\mathbf{x}_{1}^{T}, \cdots, \mathbf{x}_{V}^{T}\right]^{T}-\left[\mathbf{m}_{1 k}^{T}, \cdots, \mathbf{m}_{V k}^{T}\right]^{(t+1) T}\right)^{T} D_{i}^{(t)}\left(\left[\mathbf{x}_{1}^{T}, \cdots, \mathbf{x}_{V}^{T}\right]^{T}-\left[\mathbf{m}_{1 k}^{T}, \cdots, \mathbf{m}_{V k}^{T}\right]^{(t+1) T}\right) \\
& \leqslant \sum_{i=1}^{N_{k}}\left(\left[\mathbf{x}_{1}^{T}, \cdots, \mathbf{x}_{V}^{T}\right]^{T}-\left[\mathbf{m}_{1 k}^{T}, \cdots, \mathbf{m}_{V k}^{T}\right]^{(t) T}\right)^{T} D_{i}^{(t)}\left(\left[\mathbf{x}_{1}^{T}, \cdots, \mathbf{x}_{V}^{T}\right]^{T}-\left[\mathbf{m}_{1 k}^{T}, \cdots, \mathbf{m}_{V k}^{T}\right]^{(t) T}\right) \\
& =N_{k} f(t) .
\end{aligned}
$$

Taking into account both Lemma A.2 and (26), we get the conclusion that $f(t+1) \leqslant$ $f(t)$. It means that the objective value of (6) is descending with increased optimization iterations. Moreover, $f(t+1)=f(t)$ means that $\left(m_{k}\right)^{(t+1)}=\left(m_{k}\right)^{(t)}$. As a result, $\mathbf{m}_{k}^{(t)}=\arg \min _{\mathbf{m}_{k}} \sum_{i=1}^{N_{k}}\left(\left[\mathbf{x}_{1}^{T}, \cdots, \mathbf{x}_{V}^{T}\right]^{T}-\left[\mathbf{m}_{1 k}^{T}, \cdots, \mathbf{m}_{V k}^{T}\right]^{(t) T}\right)^{T} D_{i}^{(t)}\left(\left[\mathbf{x}_{1}^{T}, \cdots, \mathbf{x}_{V}^{T}\right]^{T}-\left[\mathbf{m}_{1 k}^{T}, \cdots, \mathbf{m}_{V k}^{T}\right]^{(t) T}\right)$,

To conclude, $\mathbf{m}_{k}^{*}=\mathbf{m}_{k}^{(t)}$.

\section{REFERENCES}

Krizhevsky Alex, Ilya Sutskever, and Geoffrey E Hinton. 2012. ImageNet Classification with Deep Convolutional Neural Networks. In Advances in Neural Information Processing Systems. 1097-1105. 
Galen Andrew, Raman Arora, Jeff Bilmes, and Karen Livescu. 2013. Deep canonical correlation analysis. In International Conference on Machine Learning. 1239-1247.

M. Van Breukelen, R. P. W. Duin, D. M. J. Tax, and J. E. Den Hartog. 1998. Handwritten Digit Recognition by Combined Classifiers. Kybernetika -Praha- 34, 4 (1998), 381-386.

Arthur E. Bryson, Yu Chi Ho, and George M. Siouris. 2007. Applied Optimal Control: Optimzation, Estimation, and Control. IEEE Transactions on Systems Man and Cybernetics 9, 6 (2007), 366-367.

Bor Chun Chen, Chu Song Chen, and Winston H. Hsu. 2015. Face Recognition and Retrieval Using CrossAge Reference Coding With Cross-Age Celebrity Dataset. IEEE Transactions on Multimedia 17, 6 (2015), 804-815.

W Chu and S Keerthi. 2007. Support Vector Ordinal Regression. Neural Computation 19, 3 (2007), $792-815$.

Hardoon David and Taylor John Shawe. 2011. Sparse Canonical Correlation Analysis. Machine Learning 83, 3 (2011), 331-353.

Lin Feng, Laihang Yu, Hai Zhu, Lin Feng, Laihang Yu, Hai Zhu, Lin Feng, Laihang Yu, and Hai Zhu. 2017. Spectral embedding-based multiview features fusion for content-based image retrieval. Journal of Electronic Imaging 26, 5 (2017), 1.

Mohammad Haghighat, Mohamed Abdel-Mottaleb, and Wadee Alhalabi. 2016. Discriminant Correlation Analysis: Real-Time Feature Level Fusion for Multimodal Biometric Recognition. IEEE Transactions on Information Forensics and Security 11, 9 (2016), 1984-1996.

Yahong Han, Fei Wu, Dacheng Tao, Jian Shao, Yueting Zhuang, and Jianmin Jiang. 2012. Sparse Unsupervised Dimensionality Reduction for Multiple View Data. IEEE Transactions on Circuits and Systems for Video Technology 22, 10 (2012), 1485-1496.

X. He and P. Niyogi. 2003. Locality preserving projections. Advances in Neural Information Processing Systems 16, 1 (2003), 186-197.

L. Hoegaerts, J. A. K. Suykens, J. Vandewalle, and B. De Moor. 2005. Subset based least squares subspace regression in RKHS. Neurocomputing 63 (2005), 293-323.

Chenping Hou, Changshui Zhang, Yi Wu, and Feiping Nie. 2010. Multiple view semi-supervised dimensionality reduction. Pattern Recognition 43, 3 (2010), 720-730.

Hua Huang, Huiting He, Xin Fan, and Junping Zhang. 2010. Super-resolution of human face image using canonical correlation analysis. Pattern Recognition 43, 7 (2010), 2532-2543.

Hongkun Ji, Xiaobo Shen, Quansen Sun, and Zexuan Ji. 2015. Sparse Discrimination based Multiset Canonical Correlation Analysis for Multi-Feature Fusion and Recognition. In British Machine Vision Conference. 141.1-141.9.

Chie Kamada, Asako Kanezaki, and Tatsuya Harada. 2015. Probabilistic Semi-Canonical Correlation Analysis. In ACM International Conference on Multimedia. 1131-1134.

Meina Kan, Shan Shiguang, Zhang Haihong, Lao Shihong, and Chen Xilin. 2015. Multi-View Discriminant Analysis. IEEE Transactions on Pattern Analysis and Machine Intelligence 38, 1 (2015), 188 - 194.

T. Kawashima, T. Ogawa, and M. Haseyama. 2013. A rating prediction method for e-commerce application using ordinal regression based on LDA with multi-modal features. In Consumer Electronics. 260-261.

J. R Kettenring. 1971. Canonical Analysis of Several Sets of Variables. Biometrika 58, 3 (1971), 433-451.

Andrzej Kijko. 1994. Seismological outliers: L1 or adaptive Lp norm application. Bulletin of the Seismological Society of America 84, 2 (1994), 473-477.

Marius Kloft, Ulf Brefeld, S?ren Sonnenburg, Pavel Laskov, Klaus Robert Mller, and Alexander Zien. 2009. Efficient and Accurate Lp-Norm Multiple Kernel Learning. In Advances in Neural Information Processing Systems. 997-1005.

Marius Kloft, Ulf Brefeld, S?ren Sonnenburg, and Alexander Zien. 2011. lp-Norm Multiple Kernel Learning. Journal of Machine Learning Research 12 (2011), 953-997.

Takumi Kobayashi and Nobuyuki Otsu. 2008. Image Feature Extraction Using Gradient Local AutoCorrelations. In Computer Vision - ECCV 2008, European Conference on Computer Vision, Marseille, France, October 12-18, 2008, Proceedings. 346-358.

Lee Kuang-Chih, Ho Jeffrey, and David J Kriegman. 2005. Acquiring linear subspaces for face recognition under variable lighting. IEEE Transactions on Pattern Analysis and Machine Intelligence 27, 5 (2005), 684-698.

Pei Ling Lai and Colin Fyfe. 2000. Kernel and Nonlinear Canonical Correlation Analysis. International Journal of Neural Systems 10, 5 (2000), 365-377.

Craig T. Lawrence. 2000. A Computationally Efficient Feasible Sequential Quadratic Programming Algorith$m$. Society for Industrial and Applied Mathematics. 1092-1118 pages. 
Thomas Melzer, Michael Reiter, and Horst Bischof. 2003. Appearance models based on kernel canonical correlation analysis. Pattern Recognition 36, 9 (2003), 1961-1971.

Feiping Nie, Heng Huang, Xiao Cai, and Chris Ding. 2010. Efficient and robust feature selection via joint 12,1-norms minimization. In International Conference on Neural Information Processing Systems. 18131821.

Elena Parkhomenko. 2008. Sparse Canonical Correlation Analysis. Statistical Applications in Genetics and Molecular Biology 8, 1 (2008), 1.

E. S. Pearson. 1936. RELATIONS BETWEEN TWO SETS OF VARIATES. Biometrika 28, 3/4 (1936), 321377.

Loki Radoslav. 2006. Sequential Quadratic Programming. Springer US. 523-560 pages.

Jan Rupnik and Marko Grobelnik. 2008. Cross-lingual search over 22 european languages. In International ACM SIGIR Conference on Research and Development in Information Retrieval. 883-883.

M. E. Sargin, E. Erzin, Y. Yemez, and A. M. Tekalp. 2006. Multimodal Speaker Identification Using Canonical Correlation Analysis. In IEEE International Conference on Acoustics, Speech and Signal Processing, 2006. ICASSP 2006 Proceedings. 613-616.

Mehmet Emre Sargin, Ycel Yemez, Engin Erzin, and A. Murat Tekalp. 2007. Audiovisual Synchronization and Fusion Using Canonical Correlation Analysis. IEEE Transactions on Multimedia 9, 7 (2007), 13961403.

Zhongrong Shi, Sheng Wang, and Chuancai Liu. 2017. Robust and sparse canonical correlation analysis based L2,p-norm. Journal of Engineering 1, 1 (2017), 1-3.

Karen Simonyan and Andrew Zisserman. 2014. Very Deep Convolutional Networks for Large-Scale Image Recognition. Computer Science (2014), 1-14.

Suzanne Weaver Smith and Christopher A. Beattie. 2015. Secant-method adjustment for structural models. Aiaa Journal 29, 1 (2015), 119-126.

Tingkai Sun, Songcan Chen, Jingyu Yang, and Pengfei Shi. 2008b. A Novel Method of Combined Feature Extraction for Recognition. In Eighth IEEE International Conference on Data Mining. 1043-1048.

Ting Kai Sun, Song Can Chen, Zhong Jin, and Jing Yu Yang. 2008a. Kernelized discriminative canonical correlation analysis. In International Conference on Wavelet Analysis and Pattern Recognition. 12831287.

Kim Tae-Kyun and Cipolla Roberto. 2009. anonical Correlation Analysis of Video Volume Tensors for Action Categorization and Detection. IEEE Transactions on Pattern Analysis and Machine Intelligence 31, 8 (2009), 1415-1428.

Qing Tian, Songcan Chen, and Lishan Qiao. 2016a. Ordinal margin metric learning and its extension for cross-distribution image data. Information Sciences 349 (2016), 50-64.

Qing Tian, Hui Xue, and Lishan Qiao. 2016b. Human Age Estimation by Considering both the Ordinality and Similarity of Ages. Neural Processing Letters 43, 2 (2016), 1-17.

Qing Tian, Wenqiang Zhang, Liping Wang, Songcan Chen, and Hujun Yin. 2018. Robust Ordinal Regression Induced by lp-Centroid. Neurocomputing 313 (2018), 184-195.

Ahonen Timo, Hadid Abdenour, and PietikoInen Matti. 2006. Face description with local binary patterns: application to face recognition. IEEE Transactions on Pattern Analysis and Machine Intelligence 28, 12 (2006), 2037-2041.

Alexei Vinokourov, John Shawe-Taylor, and Nello Cristianini. 2002. Inferring a Semantic Representation of Text via Cross-Language Correlation Analysis. Advances of Neural Information Processing Systems (2002), 1497-1504.

Liping Wang and Songcan Chen. 2017. Joint representation classification for collective face recognition . Pattern Recognition 63, 5 (2017), 182-192.

Liping Xie, Dacheng Tao, and Haikun Wei. 2016. Joint Structured Sparsity Regularized Multiview Dimension Reduction for Video-Based Facial Expression Recognition. ACM Transactions on Intelligent Systems and Technology 8, 2 (2016), 1-21.

Deming Zhai, Chang Hong, Shiguang Shan, Xilin Chen, and Gao Wen. 2012. Multiview Metric Learning with Global Consistency and Local Smoothness. ACM Transactions on Intelligent Systems and Technology 3, 3 (2012), 1-22.

Desheng Zhang, He Tian, and Zhang Fan. 2017. Real-Time Human Mobility Modeling with Multi-View Learning. ACM Transactions on Intelligent Systems and Technology 9, 3 (2017), 1-25.

Hangxing Zhou and Songcan Chen. 2014. Ordinal Discriminative Canonical Correlation Analysis. Journal of Software 25, 9 (2014), 2018-2025. 


\section{Online Appendix to: \\ Moment-Guided Discriminative Manifold Correlation Learning On Ordinal Data}

QING TIAN, WENQIANG ZHANG and MENG CAO, Nanjing University of Information Science and Technology

LIPING WANG and SONGCAN CHEN, Nanjing University of Aeronautics and Astronautics

HUJUN YIN, The University of Manchester

(c) 2010 Copyright held by the owner/author(s). 2157-6904/2010/03-ART39 $\$ 15.00$ DOI : 0000001.0000001 